\title{
Distributed Algorithm of Connected Dominating Sets Based on Energy Balance
}

\author{
Zou Dan ${ }^{1}$ \\ 1.College of mechatronic engineering, Yunnan open University, Kunming ,China
}

Key words: Wireless sensor network, energy balance, energy aware, uneven routing

\begin{abstract}
When conventional algorithm mainly constructs CDS from a perspective of CDS scale, network energy balance issue will be neglected, making some nodes of oversize residual energy become dominating ones. Due to ultra-low energy, these dominating nodes will become inactive very soon, impacting network coverage and life cycle. For this purpose, in this article, from a perspective of energy balance, construction of distributed algorithm ECDSD of minimum CDS is presented. In ECDSD algorithm, firstly, all nodes are in original state; when certain node plays a role of launching node, becomes dominating node, sends and receives message, it is decided to be a dominating or dominated node in accordance with its weight; then different message is sent in accordance with its different state, which is cycled in this way until overall network is converged so as to form CDS. Simulation result shows ECDSD algorithm presented effectively enhances network coverage rate and extends life cycle.
\end{abstract}

\section{Introduction}

A large number of sensing nodes are deployed in region of interest FOI so as to collect data in FOI. These sensing nodes are formed into wireless sensor network WSN. Each sensing node is of sensing and communication capacity. Sensing node collects original data from setting, handles and transmits data to destination. To be able to collect data in FOI without dead zone in a more all-round way, FOI coverage must be maximized. Due to interference and energy exhaustion, sensing node may be unable to work, namely inactive. Thus, enhancement of coverage rate becomes research focus for WSN [1-4].

Coverage can be divided to be dotted and regional. In regional coverage, observation area is covered by a series of sensing nodes. Dotted coverage means each dot is covered by sensing node. Coverage rate is required to be kept for both dotted and regional coverage, even in a setting where sensing node is inactive. In intensive WSN, it is very useful to keep coverage rate, reserve energy and enlarge network life by reasonably selecting a group of active sensing nodes. These active nodes effectively form connection channel for transmitting data packet to destination, which enhances both energy efficiency of nodes and coverage rate.

Topology control based on CDS (Connected Dominating Sets) is one of the most effective algorithms which enhance WSN coverage rate. Available Figure $G=(V, E)$ describes WSN in a formalized way, where $V$ is sensing nodes set and $E$ is network link set. One dominating set DS in Figure $G$ is subset $D \subseteq V$, meeting $v \in D$ or making neighboring node of $v$ in $D$ for random $\operatorname{dot} v \in V$. In case Figure exported from $D$ is connected, $D$ is called connected dominating set CDS. If $v \in C D S$ is active, such node $v$ is Dominator; otherwise, it will be called Dominatee.

To enhance energy utilization rate and data transmission efficiency, CDS is widely applied to forming route of virtual backbone network. Obviously, the smaller the number of nodes in CDS is, the higher the transmission efficiency and energy-saving efficiency will be. However, in given graph, it is a NP issue to seek minimum CDS; only suboptimal solution can be calculated.

Conventional CDS algorithm of minimum construction is mainly divided into two types, centralized and distributed. In centralized algorithm, one node undertakes and collects information about all nodes in whole network to construct minimum CDS from an overall perspective, which undoubtedly increases communication overhead and does not apply to large WSN. Distributed algorithm requires local information about node only; connection information about each node is 
estimated independently.

\section{Constraints}

Assume isomorphic WSN has $\mathrm{N}$ sensing node(s), which is (are) deployed in observation area A. Original energy of each sensing node is $\mathrm{E}$; communication radius is $R_{c}$; sensing radius is $R_{s}$; and $R_{c} \geq R_{s}$. In all sensing nodes, $R_{c}$ and $R_{s}$ are the same. Sensing area of sensing node is circular with its geographical location as center and $R_{s}$ as radius. Communication area is circular with its geographical location as center and $R_{c}$ as radius. Additionally, identifiers referenced in this article are as shown below.

Definition 1: Coverage: in 2-dimensional plane domain A, sensing node set $S=\left\{S_{1}, s_{2}, \cdots, s_{n}\right\}$ is given. Position coordinates of sensing node $s_{i}(i=1,2, \cdots, n)$ is $\left(x_{i}, y_{i}\right)$. If $\operatorname{random} \operatorname{dot}^{x \in A\left(s_{i}\right)}, x$ is covered by ${ }^{s_{i}}$.

Definition 2: Dominating set: Subset $D$ in undirected graph ${ }^{G=(V, E)}, D \neq \phi$. If there is node adjacent to $u$ in subset $D$ for $v \in V-D, D$ meeting this requirement will be called a dominating set[11] of Figure G.

\section{ECDSD algorithm}

Weight

Each sensing node broadcasts information packet to its neighboring periodically, including information about residual energy. To reduce data packet collision rate, back-off time is randomly set for each node; after timekeeping is finished, information packet will be broadcasted. Once information packet is received from neighboring node, WCC (Weighted Coverage Cost) of node is calculated.

$$
E_{\text {tot }}\left(s_{i}\right)=\sum_{\forall x \in C R\left(s_{i}\right)} E_{r}(x), \text { if } E_{r}(x)>E_{t h}
$$

Where $E_{\text {tot }}\left(s_{i}\right)$ is a neighboring node of node ${ }^{s_{i}}$, residual energy $E_{r}(x)$ is larger than energy of all nodes of threshold value ${ }^{E_{t h}}$,

WCC of sensing node ${ }^{S_{i}}$ :

$$
W C C\left(s_{i}\right)=\left\{\begin{array}{lr}
0, & \text { if } E_{r}\left(s_{i}\right)<E_{t h} \\
E_{r}\left(s_{i}\right)+E_{\text {tot }}\left(s_{i}\right), & \text { otherwise }
\end{array}\right.
$$

It can be known from formula (2) that WCC of node reflects energy level of node and neighboring node. To reduce nodes which become inactive due to energy exhaustion, WCC of node where $E_{r}\left(s_{i}\right)$ is lower than threshold value $E_{t h}$ is set as zero. In case of ultra-low residual energy, working hours will be definitely short and there will be no need to be added to CDS.

As shown in Figure 1, five sensing nodes, A, B, C, D and E, are distributed in network area, and covered and overlapped. Assume their original energy is 5J, Figure 1(a) describes communication radius and sensing radius of node; dashed circle means communication area; circle of continuous line means sensing area; Figure 1(b) marks residual energy of each node; for example, residual energy of node C is 2 and residual energy of node B is 3 . Assume $E_{t h}=1$, WCC of each node can be calculated in accordance with formula (2). For example, sensing areas of nodes A and B, C, D are overlapped; residual energies of these three nodes are all larger than threshold value; thus, $\quad W C C(A)=E_{r}(A)+E_{t o t}(A)=4+3+2+1=10 \quad$. Similarly, $\quad W C C(B)=4+3=7 \quad, \quad W C C(C)=4+2=6$, $W C C(D)=4+1=5, \quad W C C(E)=2$. 


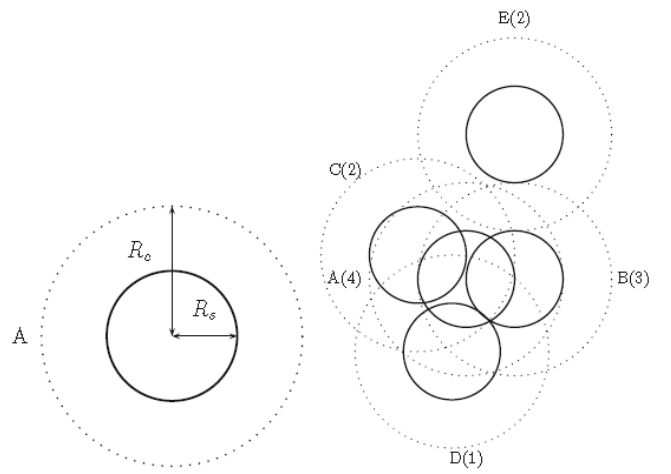

(a) Communication radius and sensing radius (b) residual energy

Figure 1 Calculation Example for WCC

\section{CDS construction process}

Core issue of ECDSD algorithm lies in how to select next dominating node by means of WCC of node, namely strategy of dominating node selection. For the purpose of brief description, assume a start from node $i$. Firstly, all nodes are in original state and marked white. Node $v$ of largest weight is sought from information packet sent by node $i$ in accordance with its single-hop neighboring node; ${ }^{W C C}(v)$ of node $v$ is larger than its own $W C C(i)$, node $v$ externally broadcasts Mes_Dominator and declares it as dominating node; otherwise, node $i$ externally broadcasts Mes_Dominator and declares it as dominating node.

As shown in Figure 2, firstly, each node is white; node 14 in Figure means node ${ }^{i}$, namely $i=$ 14. For the purpose of brief description, weight of each node is ID of node. Since weight of node $i$ is the largest in single-hop neighboring node, it externally broadcasts Mes_Dominator and becomes dominating node; thus it is marketed black.

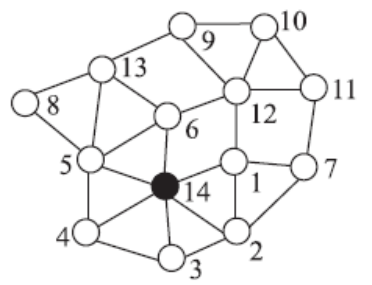

Figure 2 Occurrence of the First Dominating Node

After the first dominating node occurs, such node broadcasts Mes_Dominator. Then neighboring node receiving Mes_Dominator will become dominating node temporarily, broadcast Mes_Dominatee and be marked gray. Taking Figure 2 for example, after receiving Mes_Dominator, neighboring nodes 1, 2, 3, 4, 5 and 6 will become dominated ones and be marked gray, as shown in Figure 3.

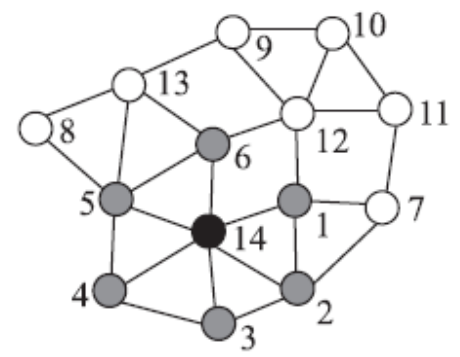

Figure 3 Occurrence of Dominated Nodes (First Round)

Once white node receives Mes_Dominatee, it will broadcast its own information packet. For example, in Figure 3, white nodes 8, 13, 12 and 7 will broadcast their information packets. Thus, gray nodes will receive information packet broadcasted by neighboring nodes, store it in list L1 and rebroadcast list L1. For example, in Figure 3, gray node 6 receives information packets from white nodes 13 and 12, store weight and ID of nodes 13 and 12 in its list L1 and externally broadcasts L1.

Subsequently, the first dominating node, namely black node, will receive L1 sent by each gray 
node, store L1 received in L2. Black node will seek white node of large weight from L2 as the second dominating node, which is namely marked black. For example, in Figure 3, node 13 is white node of largest value in L2; thus it becomes the second dominating node.

Finally, gray node, which is of the largest weight and adjacent to the second dominating node (13) , is selected to be the third dominating node from single-hop neighboring node ${ }^{N(14)}$ in the first dominating node. It can be known from Figure 3 that adjacent nodes between nodes 14 and 13 include nodes 5 and 6 . And weight of node 6 is higher than that of node 5 . Thus, node 6 becomes the third dominating node, as shown in Figure 4. The said process is repeated until there is no white node in network. All white nodes form CDS. Still taking Figure 3 as example, dominating nodes finally formed are as shown in Figure 4; nodes 12 and 11 respectively become the fourth and fifth dominating nodes.

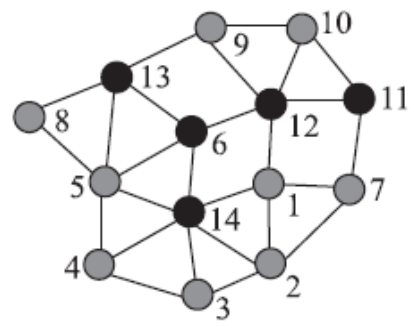

Figure 4 Occurrence of Dominating Node

\section{Performance analysis}

Simulation parameters

In this section, ECDSD algorithm is simulated. Assume $\mathrm{N}$ sensing node(s) is (are) randomly deployed in two-dimensional plane, regional area $A=200 \times 200 \mathrm{~m}^{2}$. Each simulation is independently repeated for 100 times; average will be taken as final test data.

During performance analysis process, average CDS scale, life cycle, residual energy, regional coverage rate and convergence time of CDS are selected as performance indexes. Similarly, two algorithms, EB-CDS[9] and HMCDS[10], are used for reference.

Figure 5 shows average CDS scale of three algorithms; it can be known from Figure 5 that the reason why ECDSD algorithm is slightly superior to EB-CDS and HMCDS is that ECDSD algorithm does not include node, of which energy is lower than threshold value, in CDS to reduce number of active nodes.

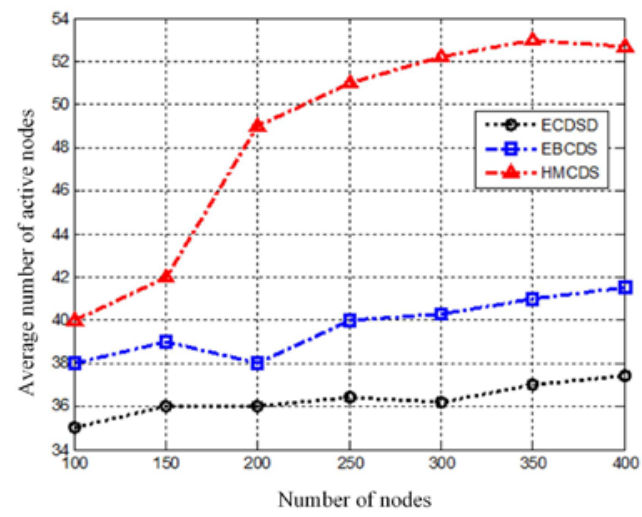

Figure 5 CDS Scale

\section{Conclusion}

In this article, distributed ECDSD algorithm of connected dominating set based on energy balance is presented. ECDSD algorithm considers energy distribution information about node. Node, where residual energy is larger than threshold value, is considered to be included in dominating set to the maximum. ECDSD algorithm selects dominating node by means of WCC; WCC includes 
energy information about sensing neighboring nodes, making network energy exhaustion more balanced. Simulation result shows ECDSD algorithm presented effectively enhances network coverage rate and extends life cycle.

\section{Reference}

[1] Kui X, Wang J, Zhang S. A data gathering algorithm based on energy-balanced connected dominating sets in wireless sensor networks[C]// Wireless Communications and NETWORKING Conference. IEEE, 2013:1139-1144.

[2] Yuanyuan Z, Xiaohua J, Yanxiang H. A distributed algorithm for constructing energy-balanced connected dominating set in wireless sensor networks[J]. International Journal of Sensor Networks, 2007, 2(1/2):68-76.

[3] Zhen-Hua W U, Fan M I, Tan B F, et al. Distributed Algorithm for Energy-Balance Minimum 2- Connected 2-Dominating Set[J]. Computer Systems \& Applications, 2014.

[4] Yuanyuan Z, Jia X, Yanxiang H. Energy efficient distributed connected dominating sets construction in wireless sensor networks[C]// International Conference on Wireless Communications and Mobile Computing. ACM, 2006:797-802.

[5] Yongsheng FU, Shanping L I, Bo Z. An Energy-Balance Connected Dominating Set Algorithm in Wireless Sensor Networks[J]. Chinese Journal of Sensors \& Actuators, 2010, 23(8):1142-1145. 